\title{
Study on Negative Poisson Ratio and Energy Absorption Characteristics of Embedded Arrow Honeycomb Structure
}

\author{
Wenzheng $\mathrm{Liu}^{1,2}$, Shiqing Huang ${ }^{1,2}$, Jiachu $\mathrm{Xu}^{1,2}$ \\ 1. School of Mechanics and Civil Engineering, Jinan University, Guangdong Province, Guangzhou City 510632, \\ China \\ 2. Key Laboratory of Major Engineering Disaster and Control, Ministry of Education, Guangzhou, 510632, \\ China \\ E-mail: 675828816@qq.com; thuangsq@jnu.edu.cn; txjch@jnu.edu.cn
}

Received: 16 July 2020; Accepted: 14 September 2020; Available online: 15 October 2020

\begin{abstract}
Impact collision exists widely in people's daily life and threatens people's life safety. Negative Poisson's ratio structure has good mechanical properties. Therefore, it is of great significance to design and study the energy absorption structure with negative Poisson's ratio effect. Based on the traditional symmetrical concave honeycomb structure (SCHS) with negative Poisson's ratio, two modified negative Poisson's ratio honeycomb structures are proposed by adding embedded straight rib arrow structure and embedded curved rib arrow structure, which are respectively called embedded straight rib arrow honeycomb structure (SRAH) and embedded curved rib arrow honeycomb structure (CRAH). Through finite element simulation experiment, the negative Poisson's ratio characteristics of two cellular cells were studied and the influence of structural parameters of the cells on the Poisson's ratio was discussed. ANSYS/LS-DYNA was used to analyze the energy absorption of the proposed three cellular structures at different impact velocities. Numerical simulation results show that the SRHS and CRAH have greater stress platform value, specific energy absorption and impact force efficiency than SCHS, indicating that the SRAH and CRAH exhibited better energy absorption efficiency and impact resistance performance.
\end{abstract}

Keywords: Negative Poisson's honeycomb; Impact resistance; Energy absorption; Finite element.

\section{Introduction}

Inspired by the natural honeycomb, researchers have created a positive Poisson's ratio honeycomb structure with the advantages of light weight, excellent energy absorption and high impact resistance, which has been widely used in many fields, including aerospace, architecture and medical. In recent years, with the emergence and development of negative Poisson's ratio materials, auxetic honeycomb material with negative Poisson's ratio have attracted numerous attentions [1-3]. The negative Poisson's ratio material exhibits lateral expansion deformation under uniaxial tension, which has higher shear modulus [4], stronger impact resistance [5] and greater indentation resistance than the traditional structure. Therefore, the negative Poisson's ratio honeycomb structure is an ideal choice for the design of deformed wings [6-10] and other aerospace structures [11-13], and the related study have attracted the attention and research of domestic and foreign scholars [14-17].

In 1982, Gibson et al.[18] firstly proposed the two-dimensional re-entrant hexagon structure, which conducted theoretical and experimental studies on its bending, elastic buckling and plastic failure, and proposed the famous classical cellular material theory (CMT). In 1991, Evans et al. [19] named negative Poisson's ratio materials as Auxetics. Since then, due to people's deeper understanding of negative Poisson's ratio, more and more scholars have studied negative Poisson's ratio structure, which greatly promoted its development [20-24]. Qiao et al. [25] studied the in-plane impact performance of the double-arrow structure at quasi-static and medium-high speeds through theory and finite element method. Compared with the traditional hexagon honeycomb structure, the reentrant hexagon honeycomb structure has better mechanical properties [26-29], but its low stiffness limits its application in reality. Therefore, it is difficult for the new structure to increase the stiffness of hexagonal honeycomb and ensure that auxetics will not be greatly reduced. Li et al.[30] proposed that the hierarchical structure was to replace each vertex of a re-entrant hexagonal structure of lower hierarchy order with a smaller reentrant hexagon, and analyzed the Poisson's ratio and energy absorption performance of the first-order and secondorder structures with finite element analysis. Lu et al. [31] proposed a new topology concept by adding a narrow ribs into the unit cell of classical re-entrant structure. They compared the Young's modulus and Poisson's ratio with the analytical method and the finite element method, and found that the young's modulus of the ribbed structure was significantly higher than that of the re-entrant hexagon structure. Li et al. [32] proposed two new 2D re-entrant 
topologies respectively was constructed by adding two sinusoidal-shaped ribs into the classical re-entrant topology and extra vertical ribs, and theirs mechanical properties(Poisson's ratio and energy absorption capacity) are studied using finite element method as a function of geometric parameters. The simulation results that the Poisson ratio of the new structure changes with the change of geometric parameters, and the energy absorption capacity of the two structures is better than the traditional hexagonal honeycomb structure. Hou Xiuhui and Yin Guansheng [33] studied the energy absorption characteristics of multi-concave Angle honeycomb structures under different impact loads using DYNA, and compared the finite element simulation results with the empirical formula proposed.

In this paper, based on the traditional symmetrical concave honeycomb structure (SCHS) with negative Poisson's ratio, two new types of negative Poisson's ratio honeycomb structures are proposed. The SRAH was constructed by adding embedded straight rib arrow structure into the unit cell of classical re-entrant topology. The CRAH was made by adding embedded curved rib arrow structure into the unit cell of classical re-entrant topology. Through finite element numerical simulation experiment, the Poisson's ratio characteristics of these two embedded arrow-shaped honeycomb cells were studied by adjusting the structural parameters. At last, the energy absorption capacities of symmetrical concave honeycomb structure (SCHS), straight ribbed arrow honeycomb structure (SRAH) and curved ribbed arrow honeycomb structure (CRAH) under impact load was studied by ANSYS/LSDYNA.

\section{Structure and material design of embedded arrow honeycomb}

\subsection{Structure design}

The traditional concave hexagon cell structure is shown in Fig.1a. Its cell structure parameters are composed of length $L_{0}$, height $H_{0}$, the depth of concavity $L_{1}$, wall thickness $t$, and out of plane width $B$. The unit cell of model SRAH, as shown in Fig.1c, is constructed by adding embedded straight rib arrow structure (as shown in Fig.1b) into the unit cell of concave hexagon cell structure. Its geometry is defined by its length $L_{0}$, height $H_{0}$, the depth of concavity $L_{1}$, Narrow rib high $H_{1}$, wall thickness $t$, and out of plane width $B$.

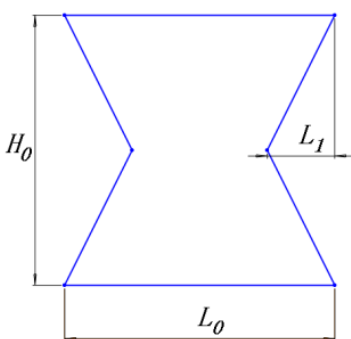

(a)

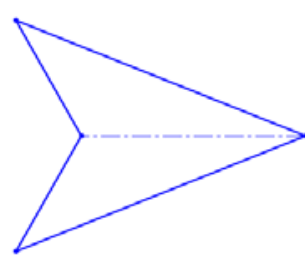

(b)

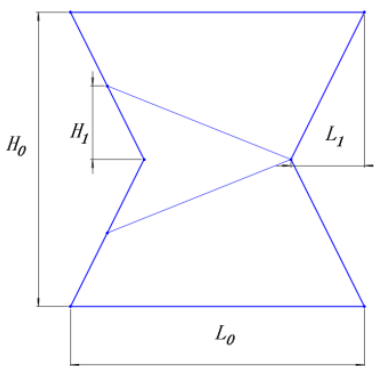

(C)

Figure 1. a) The unit cell of traditional symmetrical concave honeycomb structure. b) Straight rib arrow cell structure. c) The unit cell of embedded straight rib arrow honeycomb structure.

Analogously, the unit cell of CRAH, as shown in Fig.2b, is constructed by adding embedded curved rib arrow structure (as shown in Fig.2a) into the unit cell of concave hexagon cell structure. Its geometry is defined by its length $L_{0}$, height $H_{0}$, the depth of concavity $L_{1}$, Narrow rib high $H_{1}$, curvature of curved rib $R$, wall thickness $t$, and out of plane width $B$.

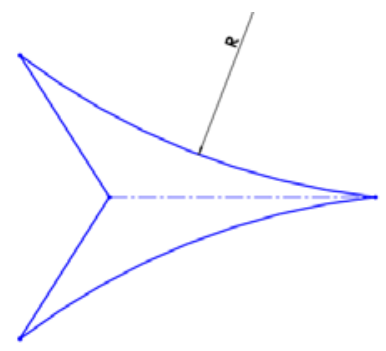

(a)

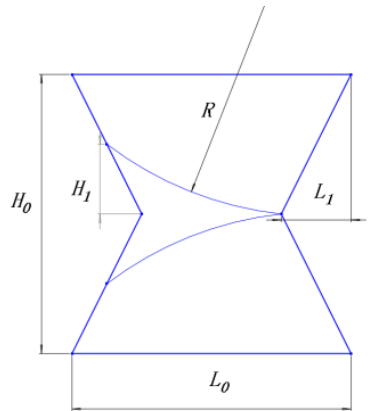

(b)

Figure 2. a) Curved rib arrow structure. b) The unit cell of embedded curved ribbed arrow honeycomb structure. 
The honeycomb structure is composed of honeycomb cells. Fig.3b shows the traditional symmetrical concave honeycomb structure (SCHS) formed by the superposition of concave hexagon cells (Fig.3a). All the structure models in this paper used for FEM simulations have eight rows and eight columns with 64 unit cells. In order to ensure the integrity of the structure, the adjacent columns are connected by ribs.

Fig.4b shows the embedded straight ribbed arrow honeycomb structure (SRAH) composed of embedded straight ribbed arrow cell (Fig.4a). Fig.5b shows the embedded curved rib arrow honeycomb structure (CRAH) composed of embedded curved rib arrow cell (Fig.5a).

For the convenience of comparative study, we define $L_{0}=H_{0}$ in the present study. Arrow parameters, $\alpha=H_{1} / H_{0}$, was defined to determine the Angle between the two narrow ribs in the structural models of SRAH and CRAH. Therefore, the change of Poisson's ratio was studied by changing the parameters $\alpha$.

SCHS cell: $L_{0}=H_{0}=20 \mathrm{~mm}, L_{1}=5 \mathrm{~mm}, t=0.7 \mathrm{~mm}, B=10 \mathrm{~mm}$

SRAH cell: $L_{0}=H_{0}=20 \mathrm{~mm}, L_{1}=5 \mathrm{~mm}, t=0.7 \mathrm{~mm}, B=10 \mathrm{~mm}$

CRAH cell: $L_{0}=H_{0}=20 \mathrm{~mm}, L_{1}=5 \mathrm{~mm}, t=0.7 \mathrm{~mm}, B=10 \mathrm{~mm}, R=0.04$

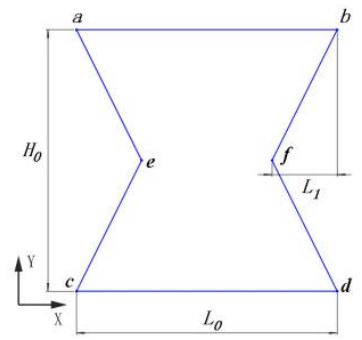

(a)

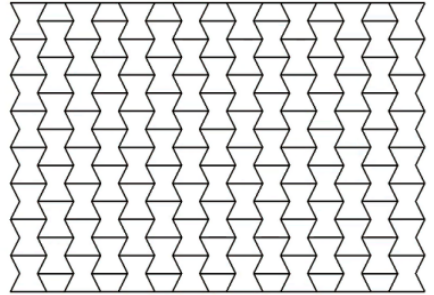

(b)

Figure 3. a) The unit cell of traditional symmetrical concave honeycomb structure. b) The traditional symmetrical concave honeycomb structure (SCHS).

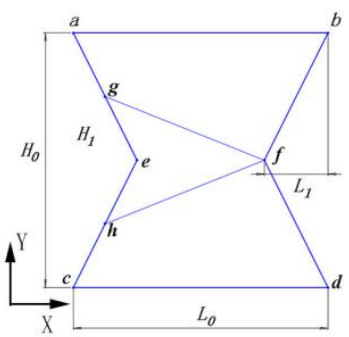

(a)

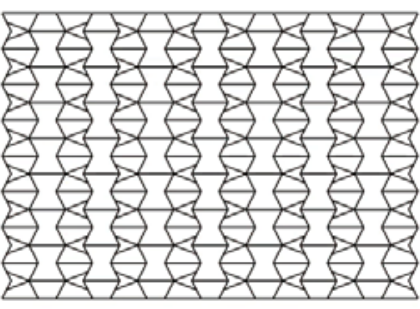

(b)

Figure 4. a) The unit cell of embedded straight ribbed arrow honeycomb structure. b) The embedded straight ribbed arrow honeycomb structure (SRAH).

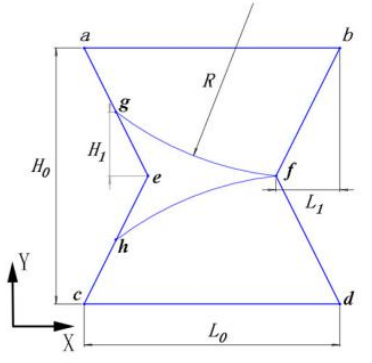

(a)

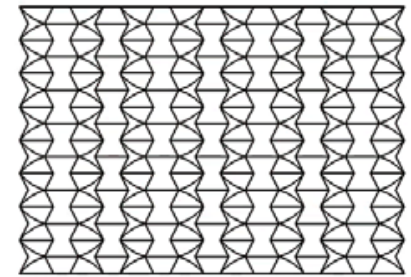

(b)

Figure 5. a) The unit cell of embedded curved ribbed arrow honeycomb structure. b) The embedded curved ribbed arrow honeycomb structure (CRAH)

\subsection{Materials and methods}

Stainless steel was selected as the material for the three structures. The material of the structure, which is given 
in Table 1, is assumed to be an ideal elastic-plastic material model. The cell wall was mashed with Shell163 elements (the number of elements depend on the edge length, and every edge had at least 5 element). The model adopts the full integral Belytschko-Tasy shell element algorithm. In order to ensure convergence, 5 integral points are defined along the thickness direction. The impact plate is assumed to be a rigid body with solid164 element. The structure model adopts the single-side automatic contact algorithm. The surface of the steel plate and the upper surface of the model are set as the face to face contact algorithm. The friction factor is set as 0.25 , and the bottom surface of the model imposes full constraints.

The Poisson's ratio was calculated from the elastic region of the stress strain diagrams obtained from the ANSYS. It is noted here that ANSYS does not take into account inertial effects and assumes time independent loading, and thus it studies the static mechanical responses. In addition, ANSYS/LS-DYNA is specially designed to study dynamic response which takes into account inertia effects.

Table 1. Material parameters

\begin{tabular}{cccccc}
\hline \multirow{2}{*}{ model } & $\begin{array}{c}\text { Young's modulus } \\
E(\mathrm{GPa})\end{array}$ & $\begin{array}{c}\text { density } \\
P\left(\mathrm{~kg} / \mathrm{m}^{3}\right)\end{array}$ & $\begin{array}{c}\text { Possion's ratio } \\
\mu\end{array}$ & $\begin{array}{c}\text { Tangent modulus } \\
E_{t}(\mathrm{Gpa})\end{array}$ & $\begin{array}{r}\text { Yield strength } \\
\sigma_{\mathrm{s}}(\mathrm{Mpa})\end{array}$ \\
\hline Rigid plate & 210 & 7800 & 0.30 & $/$ & $/$ \\
Cellular mode & 161 & 7625 & 0.29 & 1 & 402 \\
\hline
\end{tabular}

\section{Poisson's ratio analysis of cellular structure}

Poisson's ratio is defined as the ratio of transverse strain to longitudinal strain in uniaxial compression (or tension) as:

$$
v=-\frac{\varepsilon_{x}}{\varepsilon_{y}}
$$

where $v$ is Poisson's ratio, $\varepsilon_{x}$ and $\varepsilon_{y}$ are Nominal transverse positive strain and Nominal longitudinal positive strain, respectively.

$$
\varepsilon_{x}=\frac{\Delta U_{e f}}{L_{0}}
$$

where $\Delta U_{e f}$ is the relative displacement of node $\mathrm{E}$ and node $\mathrm{F}$ in the $\mathrm{X}$ direction in Fig.3a $\sim 5 \mathrm{~b}$.

$$
\varepsilon_{y}=\frac{\Delta Y}{H_{0}}
$$

where $\Delta Y$ is the displacement in $\mathrm{Y}$ direction of midpoint on plane- $a b$.

The Poisson's ratio characteristics of three kinds of cellular structures (SCHS, SRAH and CRAH), as shown in Fig.6, were analyzed by finite element simulation experiment. Stainless steel was selected as the parent material. It has a density of $7800 \mathrm{~kg} / \mathrm{m}^{3}$, Young's modulus of $161 \mathrm{Gpa}$, and Poisson's ratio of 0.29 . The static analysis module of ANSYS software is used to simulate the quasi-static compression of the model. The mesh size of the model is set to $1 \mathrm{~mm}$. The top surface of the model is applied with uniform pressure, and the bottom surface is fully fixed.

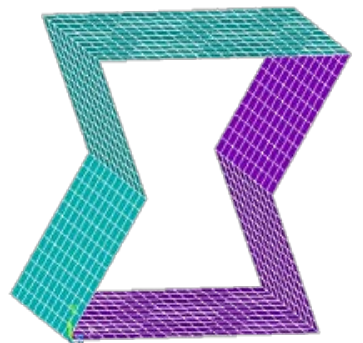

(a) SCHS cell

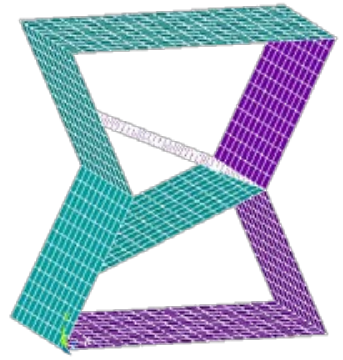

(b) SRAH cell

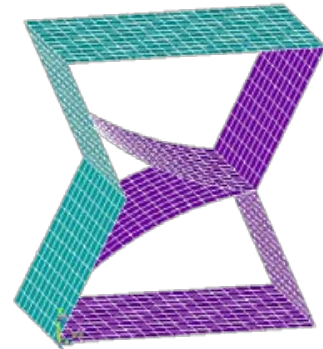

(c) CRAH cell

Figure 6. a) Finite element model of traditional symmetrical concave honeycomb structure cell. b) Finite element model of straight ribbed arrow honeycomb structure cell. c) Finite element model of curved ribbed arrow honeycomb structure. 
Three pressures of 20,50N and $100 \mathrm{~N}$ were applied to three cellular structures. The Poisson ratio of three kinds of cells under three kinds of pressure was obtained by finite element simulation and the mean value was taken. Table 2 shows the Poisson ratio of the three cellular structures when arrow parameters $\alpha=0.5$.

Table 2. Poisson's ratio of three cellular structures $(\boldsymbol{\alpha}=\mathbf{0 . 5})$

\begin{tabular}{cccc}
\hline Load cell & SCHS & SRAH & CRAH \\
\hline $20 \mathrm{~N}$ & -0.7811400 & -0.2749721 & -0.2847167 \\
$50 \mathrm{~N}$ & -0.7811437 & -0.2749727 & -0.2847177 \\
$100 \mathrm{~N}$ & -0.7811423 & -0.2749725 & -0.2847173 \\
Average & -0.7811420 & -0.2749725 & -0.2847172 \\
\hline
\end{tabular}

It can be seen from table 2 that the Poisson's ratios obtained under three different loads are very close. The average Poisson's ratio of SRAH cell and CRAH cell was -0.275 and -0.285 when $\alpha=0.5$. The Poisson ratio of SCHS cell was -0.781 , which was much higher than that of SRAH cell and CRAH cell. The reason why auxetic responses of SRAH cell and CRAH cell was much smaller than SCHS cell is that the negative Poisson's ratio effect is realized by expanding the inner concave angle and rotating the diagonal rod simultaneously. On the contrary, SRAH cell and CRAH cell resist the rotation of diagonal rod due to the function of embedded straight rib and curved rib arrow. That is consistent with literature [8].

The relationship between the Poisson's ratio of SRAH cell and CRAH cell and the arrow parameters $\alpha$ (0 $\alpha \leq 0.5$ ) is shown in Fig.7. It can be seen from Fig.7 that the Poisson's ratio of SRAH cell and CRAH cell decreased monotonically with an increasing of $\alpha$ and was close to each other.

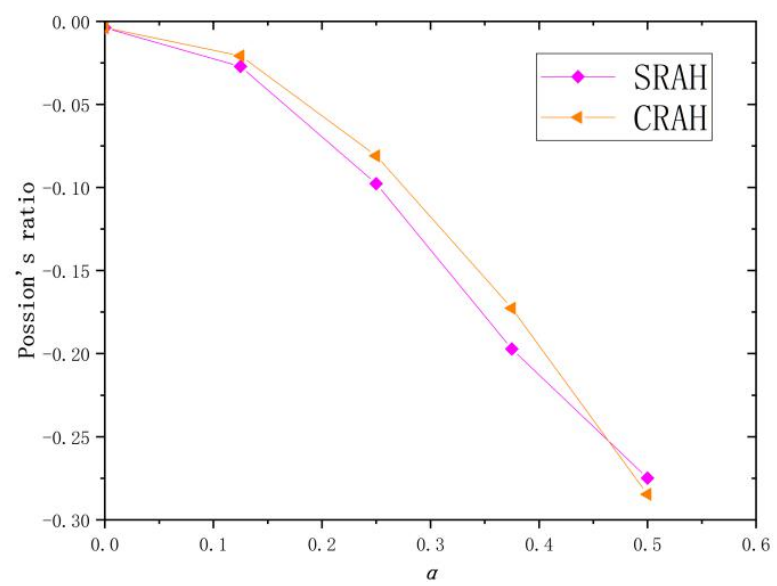

Figure 7. The relationship between the Poisson's ratio of SRAH cell and CRAH cell and $\alpha\left(\alpha=H_{1} / H_{0}\right)$.

\section{Energy absorption capacity}

\subsection{Evaluation index of energy absorption capacity}

The load-displacement curve of porous materials under impact load is generally divided into three stages: Figure 8 first shows an elastic region, followed by a platform area with almost constant load, and finally enters an elevated zone where the load rises sharply.

The nominal strain and nominal stress are defined as:

$$
\begin{aligned}
\varepsilon & =\frac{\delta}{h} \\
\sigma & =\frac{F}{A}
\end{aligned}
$$

where $\delta$ and $h$ is the compressive displacement in the $y$ direction and model of the high, respectively, $F$ is the compression reaction force of rigid plate and model, A is the cross-sectional area of contact cross section between rigid plate and model.

Total energy absorption, specific energy absorption, compressive force efficiency and platform stress are widely used to evaluate the energy absorption capacity of structures [34,35].The total energy absorption, whose magnitude is equal to the area under the load-displacement curve, represents the sum of the impact energy absorbed by the entire material from deformation to the current state. The total energy absorption is written as: 


$$
E A=\int_{0}^{\delta} P(s) d s
$$

Where $\delta$ is distance from initial state to dense state, and $P(s)$ is the instantaneous force of the impact process

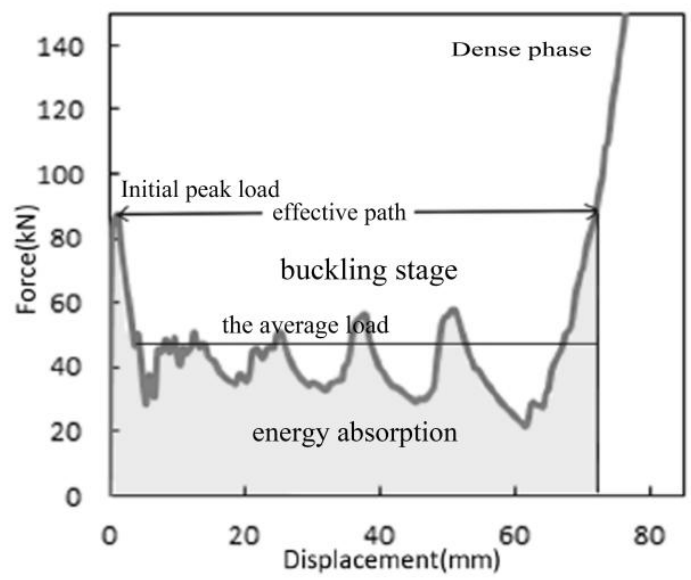

Figure 8 The load-displacement curve of porous materials

Specific absorption energy (SEA) represents the total energy absorbed by a structural material per unit mass. SEA is commonly used to evaluate the material utilization ratio of structures, and is written as:

$$
S E A=\frac{P_{m} \delta}{m}
$$

Impact efficiency (CFE), also known as load consistency index, is used to evaluate the consistency between peak load and average load. The higher the value of CFE is, the better the stability of impact process. Its formula is as follows:

$$
C F E=\frac{P_{m}}{P_{p}}
$$

where $P_{m}$ is the average of impact loads, $P_{p}$ is the initial peak load.

The platform stress values of the porous structure can be averaged from the stress values of the platform stage in the stress-strain curve, which can be expressed as:

$$
\varepsilon_{x}=\frac{\Delta U_{e f}}{L_{0}}
$$

Where $\varepsilon_{D}$ is dense strain and $\varepsilon_{y}$ is yield strain.

\subsection{Analysis of impact energy absorption characteristics of honeycomb structures}

The finite element models of the three structures under the impact of rigid plates are shown in Fig.9. In this paper, arrow parameters of SRAH and CRAH models $\alpha=0.5$. Three structural models are simulated at low speed $(3 \mathrm{~m} / \mathrm{s}$ and $6 \mathrm{~m} / \mathrm{s})$ and medium speed $(10 \mathrm{~m} / \mathrm{s}, 20 \mathrm{~m} / \mathrm{s}, 40 \mathrm{~m} / \mathrm{s}$ and $60 \mathrm{~m} / \mathrm{s})$ by ANSYS/LS-DYNA to obtain stress-strain curves.

\subsection{Deformation modal analysis}

In order to study the impact deformation of the three models at low and medium speeds, the impact velocities of $6 \mathrm{~m} / \mathrm{s}$ and $20 \mathrm{~m} / \mathrm{s}$ were selected as the representatives of low speed and medium speed respectively. The deformation modes of the three models at the impact velocity $6 \mathrm{~m} / \mathrm{s}$ is shown in Fig.10, which present that auxetic of SCHS model is the most obvious. At the initial stage of the impact process, the SCHS model has an "X-shaped" deformation pattern and obvious shear bands on the diagonal of the structure. With the increase of strain, the cells on both sides of the shear band shrink inwards until compacting. The "X-shaped" deformation mode of SRAH model and CRAH model in the initial stage of impact process is prevented due to the effect of embedded straight rib and curved rib. Small "V-shaped" deformation occurs first near the fixed end of SRAH model, and then the cells at the impact end also began to produce plastic deformation and propagated downward until the whole model 
was crushed. For the CRAH model, obvious plastic deformation first occurs at the impact end and the fixed end, and then spreads to the middle until the model collapses.

Fig.11 shows the deformation modes of the three models at the compression velocity $20 \mathrm{~m} / \mathrm{s}$. The deformation mode of SCHS model is similar to the compression velocity $6 \mathrm{~m} / \mathrm{s}$. On the contrary, the deformation modes of SRAH model and CRAH model have changed greatly. In the initial stage of impact, the cells near the impact end of SRAH model and CRAH model first occurs plastic deformation. With the impact, the plastic deformation of cell wall propagates downward until the whole model becomes dense.

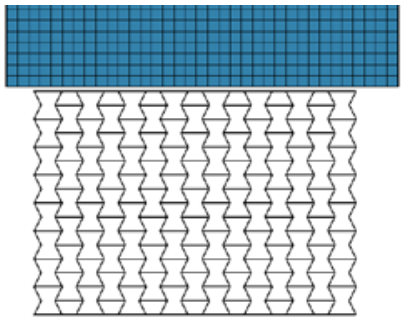

(a) SCHS

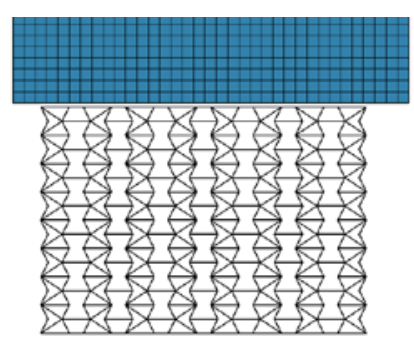

(b) SRAH

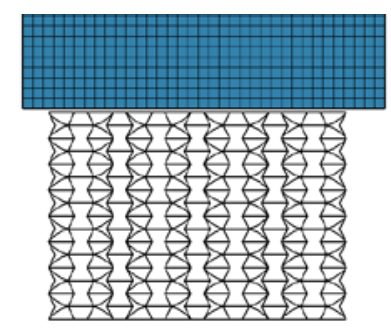

(c) $\mathrm{CRAH}$

Figure 9. a) Finite element model of traditional symmetrical concave honeycomb structure. b) Finite element model of straight ribbed arrow honeycomb structure. c) Finite element model of curved ribbed arrow honeycomb structure.
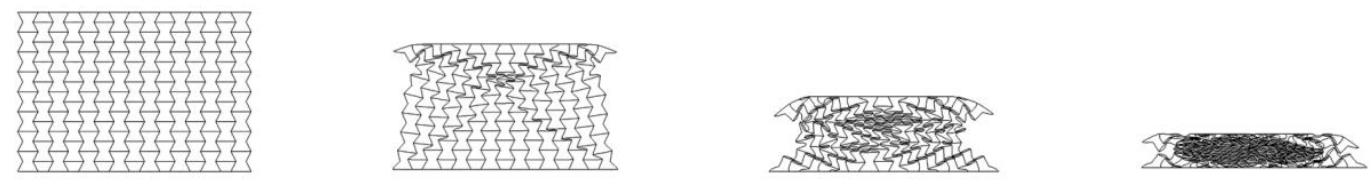

(a) SCHS
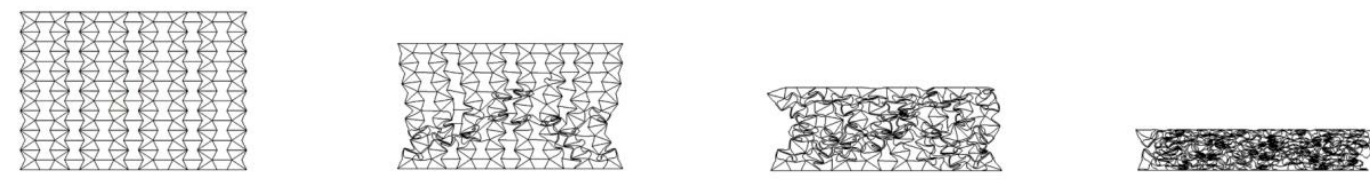

(b) SRAH
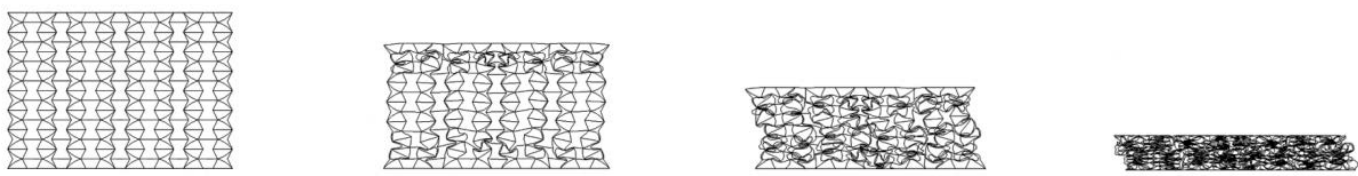

\section{(c) CRAH}

Figure 10. Deformation mechanisms. a) Traditional symmetrical concave honeycomb structure. b) Straight ribbed arrow honeycomb structure. c) Curved ribbed arrow honeycomb structure at a compression velocity $v=6 \mathrm{~m} / \mathrm{s}$.

\subsection{Energy absorption performance analysis}

Fig.12 shows the stress-strain curves of three finite element simulations at low speeds $(3 \mathrm{~m} / \mathrm{s}$ and $6 \mathrm{~m} / \mathrm{s})$. It can be seen from the figure that there is a small area with nominal stress of zero at the beginning, which is caused by the small distance between the punch and the model. Result showed that the stress peak of SCHS is lower than that of SRAH and CRAH, while the stress peak of SRAH is higher than that of CRAH. After the stress peak is reached, an oscillating stress platform region occurs due to plastic collapse deformation of the honeycomb material. The platform stress of both the SRAH and the CRAH is higher than that of the SCHS, which indicates that the cell walls of the embedded arrow structure are more difficult to collapse than the traditional concave hexagon in the plastic deformation process of the model. Therefore, the SRAH model and the CRAH model absorb more energy. 

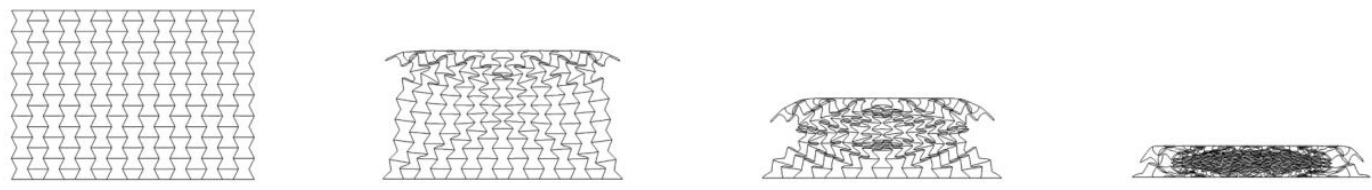

\section{(a) SCHS}
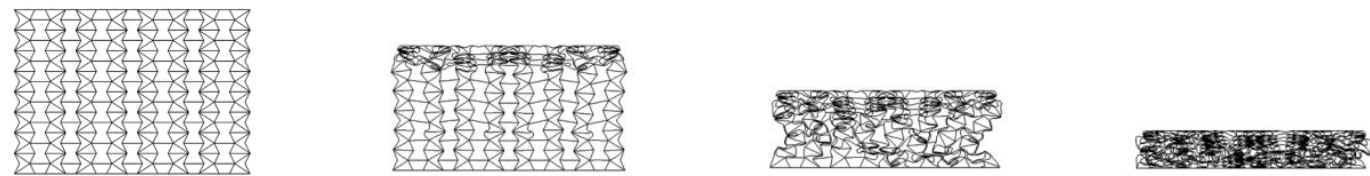

(b) SRAH
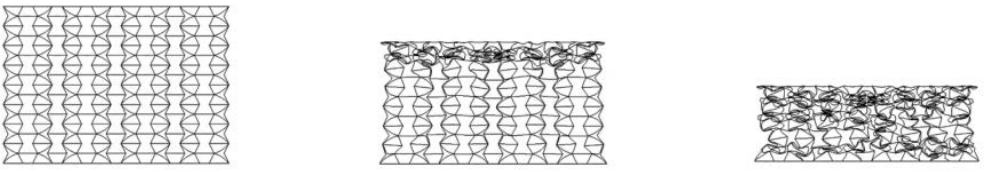

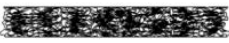

\section{(c) CRAH}

Figure 11. Deformation mechanisms. a) Traditional symmetrical concave honeycomb structure. b) Straight ribbed arrow honeycomb structure. c) Curved ribbed arrow honeycomb structure at a compression velocity $v=20 \mathrm{~m} / \mathrm{s}$.

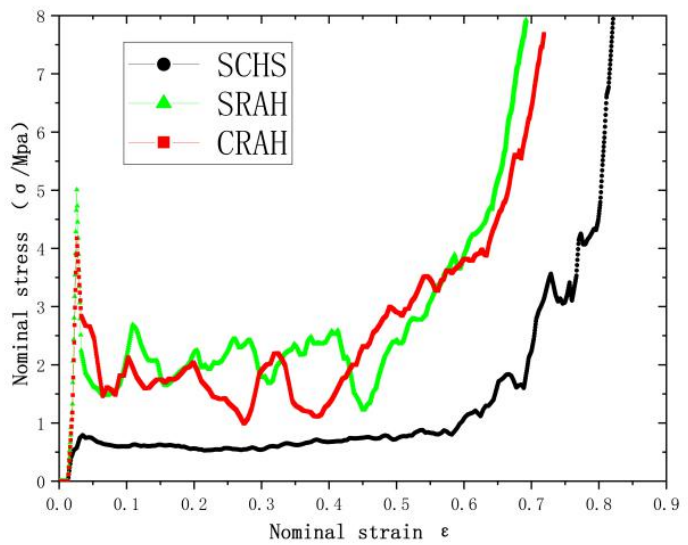

(a) striking velocity $v=3 \mathrm{~m} / \mathrm{s}$

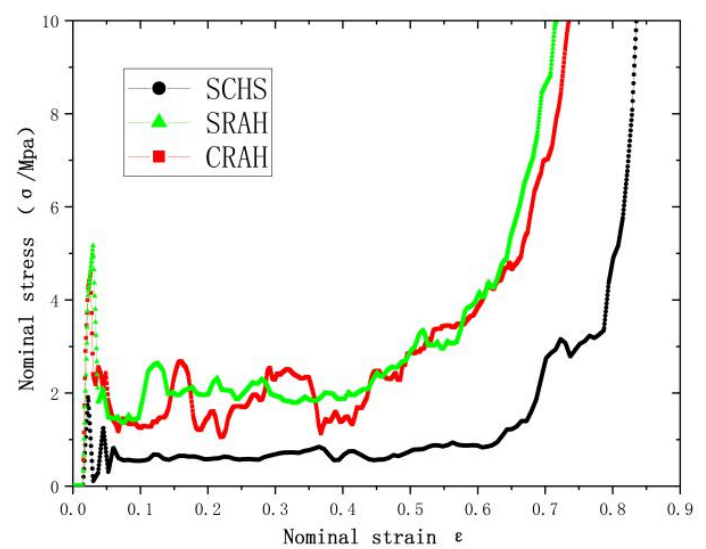

(b) striking velocity $v=6 \mathrm{~m} / \mathrm{s}$

Figure 12. The stress-strain diagrams of three models at various compression velocities. a) $v=3 \mathrm{~m} / \mathrm{s}, \mathbf{b}) v=6 \mathrm{~m} / \mathrm{s}$

Fig.13 shows the stress-strain curves of three finite element simulations at various speeds. Results showed that initial stress peak of all the models increased with an increasing compression velocity. After reaching the stress peak, the reason why the stress value of the model decreases rapidly to a low value or even close to zero that the impact end of the model is separated from the rigid plate for a short time at the high speed impact. In the platform stage, the stress-strain curve fluctuates to different degrees and the amplitude of oscillation increases with the increase of impact velocity, which is mainly caused by cell collapse and stress wave propagation in the crushing zone during the impact process. It is noted that the platform stress values of SRAH and CRAH are higher than SCHS, which indicates that the energy absorption of SRAH and CRAH is better than SCHS.

The variation curves of the platform stress of the three models under different impact velocities are shown in Fig.14a. The platform stress values of the SRAH and the CRAH are higher than the SCHS due to their deformation modes are different. After the "X-shaped" deformation of the SCHS model, the cell rapidly receded inward and the cell wall yielded. On the contrary, the narrow rib prevents the cell from recessing and the energy absorbed by the cell wall is transferred to the narrow rib. As a result, the platform stress of embedded arrow honeycomb structure is larger than the SCHS. Fig.14b shows the variation curves of impact efficiency of the three models 
under different impact velocities. Results showed that the impact efficiency of the three models decreases with the increase of impact velocity. It is noted that the impact efficiency of the SCHS at low speed $(v=3 \mathrm{~m} / \mathrm{s})$ is greater than the SRAH and the CRAH, but the impact efficiency of the SCHS was lower than that of the SRAH and the CRAH when the impact velocity was greater than $6 \mathrm{~m} / \mathrm{s}$. This indicates that the SRAH and the CRAH have higher impact resistance than the SCHS for higher impact velocity.

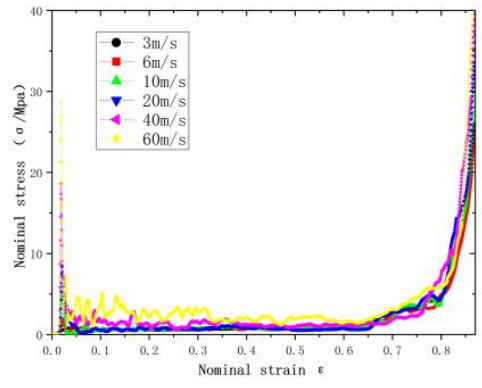

(a) SCHS

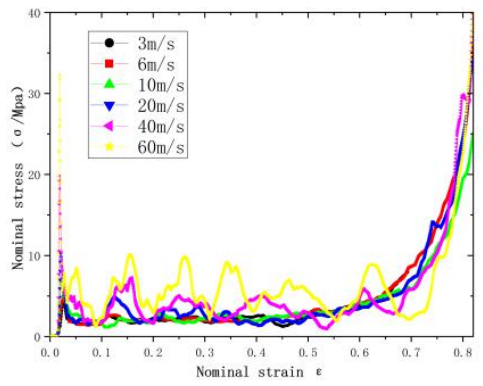

(b) SRAH

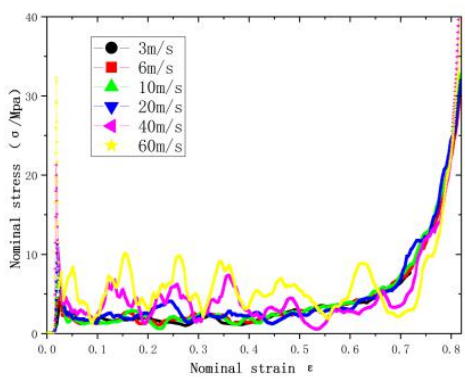

(c) CRAH

Figure 13. The stress-strain diagrams of three models at various compression velocities. a) Traditional symmetrical concave honeycomb structure. b) Straight ribbed arrow honeycomb structure. c) Curved ribbed arrow honeycomb structure.

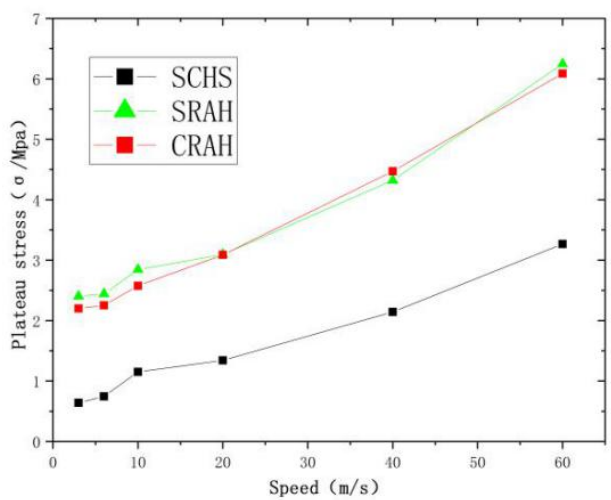

(a) Plateau stress

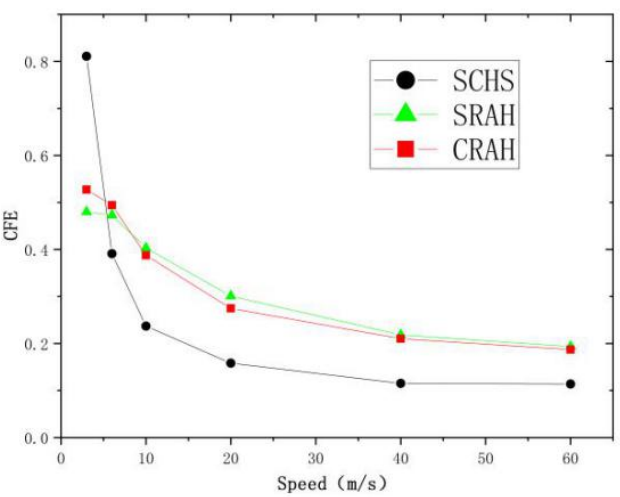

(b) CFE

Figure 14. a) The plateau stress curves of three models at various compression velocities. b) The impact efficiency curves of three models at various compression velocities.

Values of energy absorption per unit volume of the SCHS, SRAH and CRAH at different velocities are summarized in Table 3. Results showed that the energy absorption capacity of all the models increased with an increasing compression velocity. In general, the SRAH and CRAH gave rise to the highest energy absorption capacity while the SCHS exhibited the lowest energy absorption capacity. Because of the obvious auxetic effect of the SCHS, the cells deform rapidly and sink inward, which leads to the cell density without fully absorbing energy. The SRAH and CRAH extend the plastic deformation of cells due to the arrow shaped effect of embedded straight ribs and curved ribs, which makes each cell on the embedded arrow-shaped honeycomb structure reach compaction after full plastic deformation. Therefore, the energy absorption performance of the structure is improved.

Table 3. The energy absorption capacity per unit mass of three structures $(\mathrm{KJ} / \mathrm{kg})$

\begin{tabular}{cccc}
\hline & SCHS & SRAH & CRAH \\
\hline $3 \mathrm{~m} / \mathrm{s}$ & 0.41311 & 1.35253 & 1.22410 \\
$6 \mathrm{~m} / \mathrm{s}$ & 0.64187 & 1.38932 & 1.26283 \\
$10 \mathrm{~m} / \mathrm{s}$ & 1.16447 & 1.77835 & 1.56010 \\
$20 \mathrm{~m} / \mathrm{s}$ & 1.38306 & 1.97350 & 1.97307 \\
$40 \mathrm{~m} / \mathrm{s}$ & 2.28821 & 2.98515 & 3.14144 \\
$60 \mathrm{~m} / \mathrm{s}$ & 3.56720 & 4.49544 & 4.43532 \\
\hline
\end{tabular}

Comparing the platform stress, impact efficiency and specific energy absorption index of the three structures, it can be concluded that the platform stress value, impact force efficiency and specific energy absorption value of 
the SRAH and the CRAH are higher than the SCHS, which shows that the SRAH and the CRAH have better energy absorption efficiency and impact resistance than SCHS model.

\section{Conclusions}

Based on the traditional symmetrical concave honeycomb structure with negative Poisson's ratio, two new types of negative Poisson's ratio honeycomb structures are proposed by adding embedded straight rib arrow structure and embedded curved rib arrow structure. The Poisson's ratio, impact deformation mode and energy absorption efficiency of honeycomb structure with negative Poisson ratio are studied by finite element simulation. The following conclusions are obtained:

1) Under the dynamic impact, the deformation of the three honeycomb structures showed the auxetic effect, of which the SCHS model was the most obvious. The Poisson ratio of SRAH cell and CRAH cell increased with the increase of arrow parameter $\alpha$.

2) The deformation pattern of embedded arrow honeycomb structure is very different from traditional concave hexagon. The SCHS model shows an "X-shaped" deformation pattern until the whole model is crushed. On the contrary, Due to the influence of narrow ribs, the model of embedded arrow shaped honeycomb structure extends from small part of cell yield to the whole model until it is crushed.

3) With the increase of the impact velocity, the platform stress and specific energy absorption of the three models increase while the impact efficiency decreases.

4) The specific energy absorption, platform stress and impact force efficiency of SRAH model and CRAH model are higher than those of SCHS model, indicating that SRAH model and CRAH model have better energy absorption efficiency and impact resistance performance than SCHS model.

\section{References}

[1] Yang W, Li Z-M, Shi W, Xie B-H, Yang M-B. Review on auxetic materials. Journal of Materials Science. 2004; 39(10):3269-3279.

[2] Shi W, Yang W, Li Z-M, Xie B-H, Yang M-B. Advances in negative Poisson's ratio materials. Chinese Polymer Bulletin. 2003; (06):48-57. (in Chinese)

[3] Zhou L, Tan Y. Recent advance and applicaton prospects of auxetic materials. China Building Materials Science \& Technology. 2013; (05):33-34. (in Chinese)

[4] Yang Z-C, Deng Q-T. Mechanical property and application of materials and structures with negative poisson's ratio. Advances in Mechanics. 2011; 41(03):335-350. (in Chinese)

[5] Zhang X-C, Liu Y, Li N. In-plane dynamic crushing of honeycombs with negative Poisson's ratio effects. Explosion and Shock Waves. 2012; 32(05):475-482. (in Chinese)

[6] Thill C, Etches J, Bond I, Potter K, Weaver P. Morphing skins. Aeronautical Journal. 2008; 112:117-139.

[7] Evans KE. Auxetic polymers: a new range of materials. Endeavour. 1991;15(4):170-174.

[8] Masters IG, Evans KE. Models for the elastic deformation of honeycombs. J Composite Structures. 1996; 35(4).

[9] Martin J, Heyder-Bruckner J-J, Remillat C, Scarpa F, Potter K, Ruzzene M. The hexachiral prismatic wingbox concept. Physica Status Solidi (B). 2008; 245:570-577.

[10] Spadoni A, Ruzzene M, Scarpa F. Dynamic response of chiral truss-core assemblies. Journal of Intelligent Material Systems and Structures. 2006; 17:941-952.

[11] Bornengo D, Scarpa F, Remillat CD. Evaluation of hexagonal chiral structure for morphing airfoil concept. Proceedings of the Institution of Mechanical Engineers, Part G: Journal of Aerospace Engineering. 2005;219(3):185-192.

[12] Scarpa F, Burriesci G, Smith FC, Chambers B. Mechanical and electromagnetic behaviour of auxetic honeycomb structures. The Aeronautical Journal. 2003; 107 (1069):175-183.

[13] Hassan MR, Scarpa F, Ruzzene M, Mohammed N. Smart shape memory alloy chiral honeycomb. Materials Science and Engineering: A. 2008; 481:654-657.

[14] Evans K, Alderson A. Auxetic materials: Functional materials and structures from lateral thinking! Advanced Materials. 2000; 12:617-628.

[15] Lakes R. Foam structures with a negative Poisson's ratio. Science. 1987; 235:1038-1040.

[16] An W-Z. Study of the occupant crash protection of the negative Poisson's ratio seat belt webbing. Dalian University of Technology; 2013.(in Chinese)

[17] Cen S-D. Study on impact dynamics of a new auxetic honeycomb structure. Jinan University; 2018.(in Chinese)

[18] Gibson L, Ashby M, Schajer G, Robertson C. The mechanics of two-dimensional cellular materials. Proceedings of The Royal Society A: Mathematical, Physical and Engineering Sciences. 1982; 382:25-42. 
[19] Evans K, Nkansah M, Hutchinson I, Rogers S. Molecular network design. Nature. 1991; 353:124-124.

[20] Larsen U, Sigmund O, Bouwsta S. Design and fabrication of compliant micromechanisms and structures with negative Poisson's ratio. Journal of Microelectromechanical Systems. 1997; 6:99-106.

[21] Theocaris P, Stavroulakis G, Panagiotopoulos P. Negative Poisson's ratios in composites with star-shaped inclusions: A numerical homogenization approach. Archive of Applied Mechanics. 1997; 67:274-286.

[22] Lakes R. Deformation mechanisms in negative Poisson's ratio materials - Structural aspects. Journal of Materials Science. 1991; 26.

[23] Gaspar N, Ren X, Smith C, Grima J, Evans K. Novel honeycombs with auxetic behaviour. Acta Materialia. 2005; 53:2439-2445.

[24] Evans K, Nkansah MA, Hutchinson IJ. Auxetic foams: Modelling negative Poisson's ratios. Acta Metallurgica et Materialia. 1994; 42:1289-1294.

[25] Qiao J, Chen C. Analyses on the in-plane impact resistance of auxetic double arrowhead honeycombs. Journal of Applied Mechanics. 2015; 82:051007.

[26] Li D, Dong L, Yin J, Lakes R. Negative Poisson's ratio in 2D Voronoi cellular solids by biaxial compression: a numerical study. Journal of Materials Science. 2016; 51.

[27] Lakes R, Elms KJ. Indentability of conventional and negative Poisson's ratio foams. Journal of Composite Materials. 1992; 27.

[28] Chan N, Evans K. Indentation resilience of conventional and auxetic foams. Journal of Cellular Plastics. 1998; 34:231-260.

[29] Scarpa F, Tomlin PJ. On the transverse shear modulus of negative Poisson's ratio honeycomb structures. Fatigue \& Fracture of Engineering Materials \& Structures. 2000;23(8):717-720.

[30] Li D, Yin J, Dong L, Lakes R. Numerical analysis on mechanical behaviors of hierarchical cellular structures with negative Poisson's ratio. Smart Materials and Structures. 2017; 26:025014.

[31] Lu Z-X, Li X, Yang Z-Y, Xie F. Novel structure with negative Poisson's ratio and enhanced Young's modulus. Composite Structures. 2015; 138.

[32] Li D, Yin J, Dong L, Lakes RS. Strong re-entrant cellular structures with negative Poisson's ratio. Journal of Materials Science. 2018; 53(5):3493-3499.

[33] Hou X-H, Yin G-S. Dynamic crushing preformance analysis for auxetic honeycomb strycture. Journal of Mechanical Strength. 2016; 38(05):905-910.(in Chinese)

[34] Lin Z-Q. Analysis of thin-walled structure for energy absorption. South China University of Technology; 2016. (in Chinese)

[35] Fan X-G, Yin X-Y, Tao Y, Chen M-J, Fang D-N. Mechanical behavior and energy absorption of honeycomb materials under out-of-plane dynamic. Acta Mechanica Solida Sinica. 2015; 36(02):114-122.(in Chinese)

(C) 2020 by the author(s). This work is licensed under a Creative Commons Attribution 4.0 International License (http://creativecommons.org/licenses/by/4.0/). Authors retain copyright of their work, with first publication rights granted to Tech Reviews Ltd. 